\title{
Detection of circulating vascular endothelial growth factor and matrix metalloproteinase-9 in non-small cell lung cancer using Luminex multiplex technology
}

\author{
YE ZHANG ${ }^{1,2}$, JIAN-ZHONG WU ${ }^{2}$, JUN-YING ZHANG ${ }^{3}$, JING XUE ${ }^{4}$, \\ RONG MA ${ }^{2}$, HAI-XIA CAO ${ }^{2}$ and JI-FENG FENG ${ }^{1}$ \\ ${ }^{1}$ Department of Chemotherapy and ${ }^{2}$ Research Center of Clinical Oncology, The Affiliated Jiangsu Cancer Hospital, \\ Nanjing Medical University, Nanjing, Jiangsu 210009; ${ }^{3}$ Department of Oncology, Xuzhou Medical College, \\ Xuzhou, Jiangsu 221000; ${ }^{4}$ Nanjing University of Technology, Nanjing, Jiangsu 210008, P.R. China
}

Received May 20, 2013; Accepted November 13, 2013

DOI: $10.3892 / \mathrm{ol} .2013 .1718$

\begin{abstract}
It has been previously reported that vascular endothelial growth factor (VEGF) and matrix metalloproteinase (MMP)-9 are important for the occurrence and development of non-small cell lung cancer (NSCLC). The present study was designed to detect the serum levels of VEGF and MMP-9 in NSCLC, and to explore their diagnostic and prognostic values. A total of 543 cases were involved, of which 332 were NSCLC (272 cases in the pretreatment group and 60 cases in the postoperative group), 91 were patients with benign lung diseases and 120 were healthy controls. The serum levels of VEGF and MMP-9 were determined by Luminex multiplex technology. The serum levels of VEGF and MMP-9 were found to be significantly higher in the pretreatment group than those in the patients with benign lung diseases and healthy controls (VEGF, P<0.0001; MMP-9, P<0.0001). Compared with the pretreatment group, the serum levels of VEGF and MMP-9 in the postoperative group were significantly decreased (VEGF, $\mathrm{P}=0.005$; MMP-9, $\mathrm{P}=0.002$ ), and the levels of VEGF and MMP-9 in the pretreatment group of patients with stages III and IV were higher than those with stages I and II (VEGF, $\mathrm{P}<0.0001$; MMP-9, $\mathrm{P}=0.021$ ). In addition, the levels of VEGF and MMP-9 were found to closely correlate with lymph node metastasis (VEGF, $\mathrm{P}<0.0001$; MMP-9, $\mathrm{P}<0.0001$ ) in the pretreatment group, while being independent of other clinicopathological parameters $(\mathrm{P}>0.05)$. Furthermore, a positive correlation was observed between the serum levels of VEGF and MMP-9 $(\mathrm{r}=0.159 ; \mathrm{P}=0.009)$. A receiver operating
\end{abstract}

Correspondence to: Dr Ji-Feng Feng, Department of Chemotherapy, The Affiliated Jiangsu Cancer Hospital, Nanjing Medical University, 42 Baizi Ting Road, Nanjing, Jiangsu 210009, P.R. China

E-mail: zhangye881221@163.com

Key words: Luminex, vascular endothelial growth factor, matrix metalloproteinase-9, non-small cell lung cancer characteristic curve analysis showed that the diagnostic value of MMP-9 was higher than that of VEGF in the pretreatment group. The log-rank test indicated that the inoperable NSCLC patients with low levels of VEGF exhibited a significantly longer overall survival time than those with high VEGF levels $(\mathrm{P}<0.0001)$. Additionally, the serum levels of VEGF and lymph node metastasis were identified as independent prognostic factors of the inoperable NSCLC patients in a multivariate Cox regression analysis $(\mathrm{P}<0.05)$. These results indicated that VEGF and MMP-9 may be potential biomarkers for the diagnosis and prognosis of NSCLC.

\section{Introduction}

In recent decades, lung cancer has become the leading cause of cancer-related mortality in China. In 2004, 66.7/100,000 individuals succumbed to lung cancer in China (1). Non-small cell lung cancer (NSCLC) is the principal type of lung cancer, with a 5 -year survival rate of $<15 \%$ (2). Therefore, the identification of specific biomarkers associated with occurrence, development and prognosis of NSCLC is essential.

In 1971, Folkman reported that new angiogenesis maintains tumor growth when the tumor is $>2 \mathrm{~mm}$ and predicted that the presence of a tumor angiogenesis factor leads to the formation of these vessels (3). In this process, vascular endothelial growth factor (VEGF) families are the most important molecules, such as VEGF-A, -B, -C and -D and the placenta growth factor. VEGF-A, which is also called VEGF, is of great importance in angiogenesis (4).

In cancer, degradation of the extracellular matrix results in tumor cell growth, proliferation and metastasis. The matrix metalloproteinases (MMPs) represent the most critical family of endopeptidases associated with tumorigenesis. MMP-9 is a member of the MMP family and plays an evident role in the angiogenesis of tumors as well as regulating the bioavailability of VEGF (5).

The FLEXMAP 3D ${ }^{\text {TM }}$ system is a multiplex platform based on suspension assay technology. The prominent advantage of this technology is that it can detect $\leq 500$ types of analytes simultaneously in a single well. Its multiplexed function and 
enhanced sensitivity have been the cause of its wide use in recent years (6). In the present study, the serum levels of VEGF and MMP-9 were detected using Luminex multiplex technology, and their diagnostic and prognostic values were explored in NSCLC.

\section{Materials and methods}

Patients. In total, 332 cases of histopathologically confirmed NSCLC and 91 cases of confirmed benign lung disease were enrolled from the Affiliated Jiangsu Cancer Hospital, Nanjing Medical University (Nanjing, China) between February 2009 and November 2012. Of the NSCLC patients, 272 were classified as the pretreatment group and the remainders as the postoperative group. Initially, all the patients in the pretreatment group had been pathologically diagnosed with NSCLC and had not received any prior treatment. However, the patients in the postoperative group had received lung surgery in the previous month. The characteristics of the pretreatment group are shown in Table I; the median age of the patients was 61 years (range, 30-84 years) and all cases were staged according to the latest TNM staging issued in 2009 by the International Union Against Cancer. Of the 91 cases with benign lung diseases, 64 were pulmonary hamartomas, 17 were pulmonary inflammatory pseudotumor, six were pulmonary fibromas and four were pulmonary chondromas. The median age of the patients with benign lung dieseases was 42 years (range, 32-69 years). In addition, 120 healthy controls (without any abnormalities following a comprehensive examination) were enrolled, with a median age of 59 years (range, 35-79 years). A total of 155 inoperable NSCLC (stages IIIb and IV) patients were successfully followed up and the median survival time was 8 months (range, 1-20 months).

Collection and preservation of blood samples. In total, $3 \mathrm{ml}$ venous blood was extracted from the fasting patients and healthy controls. The blood samples were placed into the endotoxin- and pyrogen-free test tubes immediately. The whole blood specimens were then shaken three times and left to coagulate for $30 \mathrm{~min}$ at room temperature. Finally, the blood samples were centrifuged at $1,000 \mathrm{x} \mathrm{g}$ for $10 \mathrm{~min}$, and the serum was removed and stored at $-80^{\circ} \mathrm{C}$ prior to use. The serum of the participants was obtained following approval by the Ethics Committee of Jiangsu Cancer Hospital (Nanjing, China). Written informed consent was obtained from the patients.

Luminex multiplex technology for VEGF and MMP-9. Luminex multiplex technology was used to conduct the present study. The FLEXMAP 3D system was supplied by Luminex Corporation (Austin, TX, USA). The serum levels of VEGF and MMP-9 were determined using human cytokine/chemokine panel (cat.no. MPXHCYTO-60K) and human cardiovascular disease panel 1 (cat. no. HCVD1-67AK) from Millipore (Billerica, MA, USA), respectively. For the main immunoassay procedure for VEGF and MMP-9, all reagents were allowed to warm to room temperature $\left(20-25^{\circ} \mathrm{C}\right)$ prior to use. The placement of standards [0 (background), 3.2, 16, 80, 400, 2,000 and 10,000 pg/ml for VEGF; and 0 (background),
0.016, 0.08, 0.4, 2.0, 10.0, 50.0 ng/ml for MMP-9], controls 1 and 2 and samples on the Well Map Worksheet were then diagrammed in a vertical configuration. Subsequently, the filter plate was prewetted by pipetting $200 \mu$ l assay buffer into each well of the microtiter filter plate and was sealed and mixed on a plate shaker for $10 \mathrm{~min}$ at room temperature (20-25 $\left.{ }^{\circ} \mathrm{C}\right)$. Assay buffer was then removed by vacuum and $25 \mu \mathrm{l}$ each standard or control was added into the appropriate wells. Assay buffer was used as the $0 \mathrm{pg} / \mathrm{ml}$ standard (background); $25 \mu \mathrm{l}$ assay buffer was added to the sample well and $25 \mu \mathrm{l}$ serum matrix solution was added to the background, standard and control wells. Next, $25 \mu 1$ sample was added to the appropriate wells, the mixing bottle was vortexed and $25 \mu \mathrm{l}$ beads from the kit were added to each well. Following incubation with agitation on a plate shaker overnight at $4^{\circ} \mathrm{C}$, the samples were washed twice with $200 \mu 1$ per well of wash buffer and $25 \mu \mathrm{l}$ detection antibody was added to each well. After incubation with agitation on a plate shaker for $1 \mathrm{~h}$ at room temperature $\left(20-25^{\circ} \mathrm{C}\right), 2 \mu 1$ streptavidin-phycoerythrin was added to each well. The plate was then further incubated for $30 \mathrm{~min}$, washed twice with $200 \mu \mathrm{l} /$ well wash buffer and $150 \mu 1$ (100 $\mu$ l for MMP-9) sheath fluid was added to each well. The plate was run on the FLEXMAP $3 \mathrm{D}^{\mathrm{TM}}$ system and the median fluorescence intensity results were saved and analyzed using a weighted five-parameter logistic method for calculating the concentration of samples. The assay was performed in duplicate.

Statistical analysis. SPSS 21.0 (SPSS, Inc., Chicago, IL, USA) was used for the statistical analysis. The experimental data had a skewed distribution; therefore, non-parametric analysis was conducted. The Mann-Whitney U test was used to compare the variables between two groups, while the Kruskal-Wallis test was used to compare variables between more than two groups. The correlation between VEGF and MMP-9 was analyzed by Spearman's rank correlation. The area under the receiver operating characteristic curve (AUROC) was calculated and the logistic regression analysis was used to evaluate the diagnostic value of the two predictors. The overall survival time was calculated as the duration between the date of the initial pathological diagnosis and mortality or the latest follow-up. The median serum levels of VEGF and MMP-9 were used as the cut-off points. The overall survival rates were estimated using the Kaplan-Meier survival curves and, simultaneously, the log-rank test was used to compare the survival distributions. Univariate and multivariate Cox regression models were used to evaluate the effect of clinicopathological parameters on patient prognosis. The median and $25-75 \%$ quartiles were used for statistical description, and $\mathrm{P}<0.05$ was considered to indicate a statistically significant difference.

\section{Results}

Serum levels of VEGF and MMP-9 in the pretreatment group of NSCLC, patients with benign lung diseases and healthy controls. The concentration of VEGF and MMP-9 in the serum of the pretreatment group was significantly higher than that of the patients with benign lung diseases and the healthy controls [VEGF: $81 \mathrm{pg} / \mathrm{ml}$ (range, 47-152.5 pg/ml) vs. $59 \mathrm{pg} / \mathrm{ml}$ (range, 
Table I. Characteristics of the pretreatment group of NSCLC.

\begin{tabular}{lc}
\hline Clinicopathological characteristics & Patients, $\mathrm{n}(\%)$ \\
\hline Gender & \\
Male & $196(72.1)$ \\
Female & $76(27.9)$ \\
Age, years & \\
>60 & $159(58.5)$ \\
$\leq 60$ & $113(41.5)$ \\
Tumor location & \\
Left lung & $122(44.9)$ \\
Right lung & $148(54.4)$ \\
Whole lung & $2(0.7)$ \\
TNM stage & \\
I & $35(12.9)$ \\
II & $27(9.9)$ \\
IIIa & $32(11.8)$ \\
IIIb & $43(15.8)$ \\
IV & $135(49.6)$ \\
Lymph node metastasis & \\
Yes & $214(78.7)$ \\
No & $58(21.3)$ \\
Grading & $18(6.6)$ \\
1 & $128(47.1)$ \\
2 & $126(46.3)$ \\
3 & \\
Histological type (NSCLC) & \\
Squamous carcinoma & $190(69.9)$ \\
Adenocarcinoma & \\
Adenosquamous carcinoma & \\
Sarcoma & $(0.3)$ \\
\hline & \\
\hline &
\end{tabular}

NSCLC, non-small cell lung cancer.

21-118 pg/ml) and 62.5 pg/ml (range, 25.25-116.5 pg/ml), respectively $(\mathrm{P}<0.0001)$; and MMP-9: $1,092.5 \mathrm{ng} / \mathrm{ml}$ (range, $660.5-1,825 \mathrm{ng} / \mathrm{ml}$ ) vs. $326 \mathrm{ng} / \mathrm{ml}$ (range, 169-509 $\mathrm{ng} / \mathrm{ml}$ ) and $259 \mathrm{ng} / \mathrm{ml}$ (range, $122.25-531 \mathrm{ng} / \mathrm{ml}$ ), respectively $(\mathrm{P}<0.0001)]$. However, a statistically significant difference was not observed between the patients with benign lung diseases and the healthy controls in terms of VEGF and MMP-9 serum levels ( $\mathrm{P}>0.05)$ (Fig. 1).

Serum levels of VEGF and MMP-9 in the pretreatment and postoperative groups of NSCLC. Compared with the pretreatment group, the serum levels of VEGF and MMP-9 were significantly decreased following the pneumonectomy [VEGF: $81 \mathrm{pg} / \mathrm{ml}$ (range, 47-152.5 pg/ml) vs. $56.5 \mathrm{pg} / \mathrm{ml}$ (range, 29.75-111.75 pg/ml), respectively $(\mathrm{P}=0.005)$; and MMP-9: $1,092.5 \mathrm{ng} / \mathrm{ml}$ (range, 660.5-1,825 ng/ml) vs. $778.5 \mathrm{ng} / \mathrm{ml}$ (range, 461.75-1,266.25 ng/ml), respectively ( $\mathrm{P}=0.002)]$ (Fig. 2).

Correlation between serum levels of VEGF and MMP-9 and clinicopathological parameters in the pretreatment group of NSCLC. As shown in Table II, the concentra-
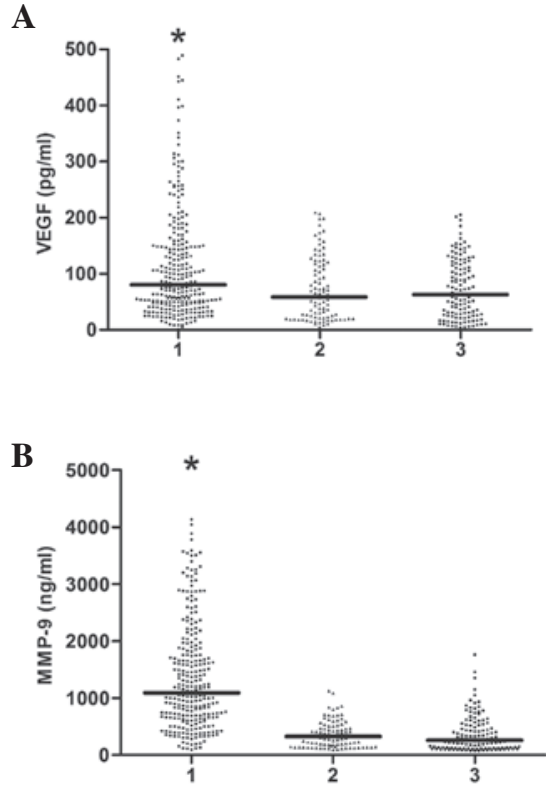

Figure 1. Serum levels of VEGF and MMP-9 in the pretreatment group of NSCLC, patients with benign lung diseases and healthy controls. Each dot represents an individual and the horizontal lines represent the median values. Expression of (A) VEGF and (B) MMP-9 in all groups. Groups are presented as follows: 1, pretreatment group of NSCLC; 2 , patients with benign lung diseases; and 3, healthy controls. ${ }^{*} \mathrm{P}<0.0001$ vs. groups 2 and 3 . VEGF, vascular endothelial growth factor; MMP-9, matrix metalloproteinase-9; NSCLC, non-small cell lung cancer.

\section{A}

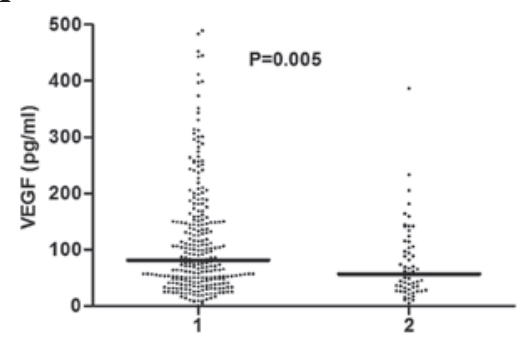

B

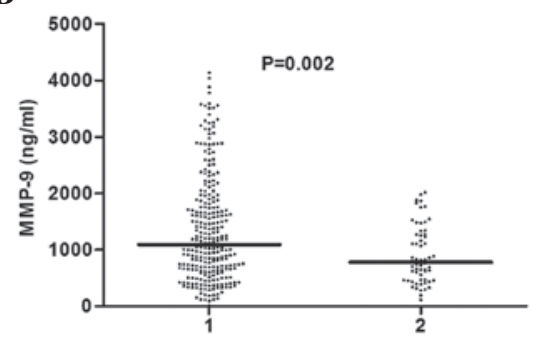

Figure 2. Serum levels of VEGF and MMP-9 in the pretreatment and postoperative groups of NSCLC. Each dot represents an individual and the horizontal lines represent the median values. Expression of (A) VEGF and (B) MMP-9 in the two groups. Groups are presented as follows: 1, pretreatment; and 2, postoperative. VEGF, vascular endothelial growth factor; MMP-9, matrix metalloproteinase-9; NSCLC, non-small cell lung cancer.

tion of VEGF in patients with TNM stages III and IV was higher than that in patients with stages I and II $[101.5 \mathrm{pg} / \mathrm{ml}$ (range, $50-183.25 \mathrm{pg} / \mathrm{ml}$ ) vs. $56.5 \mathrm{pg} / \mathrm{ml}$ (range, $34-76 \mathrm{pg} / \mathrm{ml}$ ), respectively; $\mathrm{P}<0.0001$ ] (Fig. $3 \mathrm{~A}$ ). The serum levels of VEGF were significantly higher in patients with lymph node 

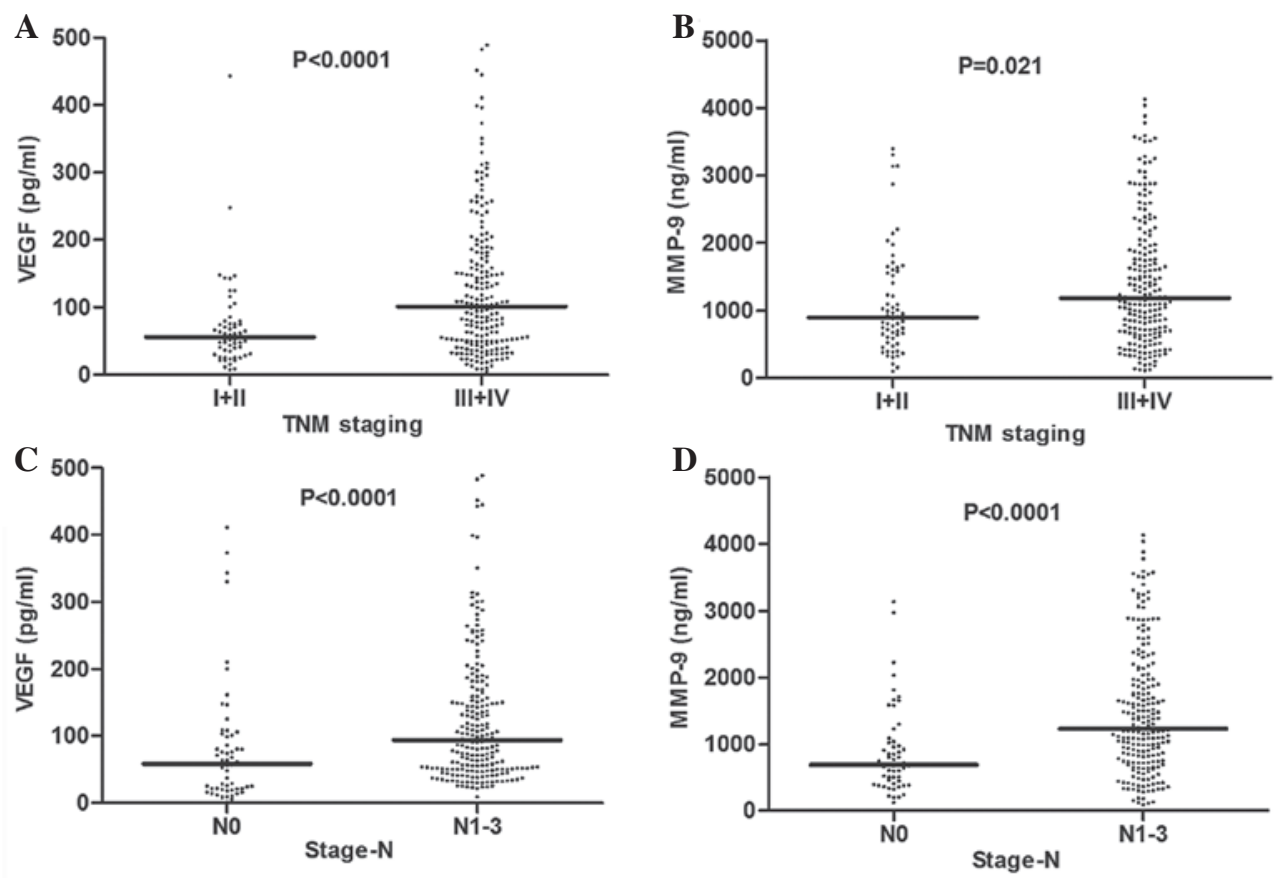

Figure 3. Differences between the serum levels of VEGF and MMP-9 in the pretreatment group of NSCLC according to TNM staging and lymph node metastasis (Stage-N). Each dot represents an individual and the horizontal lines represent the median values. Differences between (A) VEGF and (B) MMP-9 in the patients according to TNM staging and (C) VEGF and (D) MMP-9 in the patients according to lymph node metastasis. VEGF, vascular endothelial growth factor; MMP-9, matrix metalloproteinase-9; NSCLC, non-small cell lung cancer.
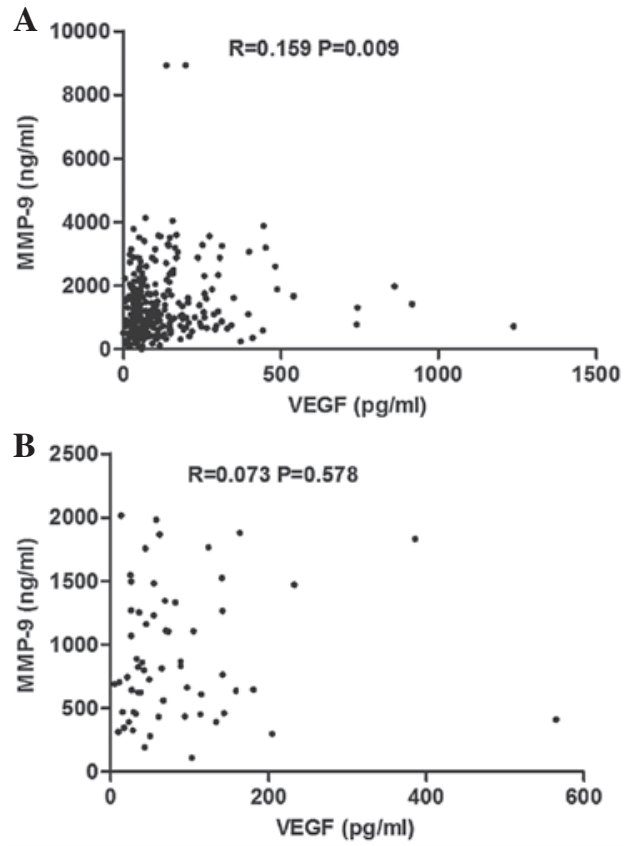

Figure 4. Scatter plot of the correlation between the serum levels of VEGF and MMP-9 in the (A) pretreatment and (B) postoperative NSCLC groups. VEGF, vascular endothelial growth factor; MMP-9, matrix metalloproteinase-9; NSCLC, non-small cell lung cancer.

metastasis compared with those without lymph node metastasis $[94 \mathrm{pg} / \mathrm{ml}$ (range, $50-170 \mathrm{pg} / \mathrm{ml}$ ) vs. $58 \mathrm{pg} / \mathrm{ml}$ (range, 20.75-101.5 pg/ml), respectively; $\mathrm{P}<0.0001$ ] (Fig. 3C). Moreover, the levels of MMP-9 in serum was found to significantly correlate with TNM staging $[1,182 \mathrm{ng} / \mathrm{ml}$ (range, $692-1,932.25 \mathrm{ng} / \mathrm{ml})$ vs. $894 \mathrm{ng} / \mathrm{ml}(572-1,586.25 \mathrm{ng} / \mathrm{ml})$, respectively; $\mathrm{P}=0.021]$ (Fig. 3B) and lymph node metastasis [1,299.5 ng/ml (range, 740.25-1,994.5 ng/ml), vs. $691.5 \mathrm{ng} / \mathrm{ml}$ (range, 404.75-1,028 ng/ml), respectively; P<0.0001] (Fig. 3D). However, no differences were identified between the serum levels of the two factors and other clinicopathological parameters $(\mathrm{P}>0.05)$.

Correlation between the serum levels of VEGF and MMP-9 in NSCLC patients. As shown in Fig. 4, Spearman's rank correlation was used to analyze the correlation between the serum levels of VEGF and MMP-9 in the pretreatment group of NSCLC. The results indicated that the expression of VEGF was found to significantly correlate with the expression of MMP-9 ( $\mathrm{r}=0.159 ; \mathrm{P}=0.009$ ) (Fig. 4A). However, no statistically significant difference was identified between the serum levels of VEGF and MMP-9 in the postoperative group ( $r=0.073$; $\mathrm{P}=0.578)$ (Fig. 4B).

Diagnostic value of VEGF and MMP-9 in the pretreatment group of NSCLC. The receiver operating characteristic curves and the logistic regression models were used to assess the diagnostic value of the serum levels of VEGF and MMP-9 in the pretreatment group of NSCLC. The AUROC of the serum levels of VEGF, differentiating the pretreatment group from the patients with benign lung diseases, was 0.632 (95\% CI, 0.566-0.698) compared with 0.885 (95\% CI, 0.852-0.919) for MMP-9. The AUROC of VEGF and MMP-9 was 0.624 (95\% CI, 0.565-0.683) and 0.88 (95\% CI, 0.845-0.914), respectively, for differentiating the pretreatment group from the healthy controls. In addition, the AUROC of VEGF for distinguishing between the pretreatment group and patients with benign lung diseases and healthy controls was 0.627 (95\% CI, 0.578-0.677), while 
Table II. Correlation between serum VEGF and MMP-9 levels and clinicopathological parameters in the NSCLC pretreatment group.

\begin{tabular}{|c|c|c|c|c|c|c|}
\hline \multirow[b]{2}{*}{ Variable } & \multicolumn{3}{|c|}{ VEGF, pg/ml } & \multicolumn{3}{|c|}{ MMP-9, ng/ml } \\
\hline & Median, $\mathrm{n}$ & Q1-Q3, n & P-value & Median, $\mathrm{n}$ & Q1-Q3, n & P-value \\
\hline \multicolumn{7}{|l|}{ Gender } \\
\hline Male & 86 & $49.25-167.75$ & 0.055 & 1159 & $693.5-1950.75$ & 0.075 \\
\hline Female & 70 & $34-131.25$ & & 939.5 & $607-1622.5$ & \\
\hline \multicolumn{7}{|l|}{ Age, years } \\
\hline$>60$ & 82 & $47-159$ & 0.981 & 1098 & $660-1758$ & 0.911 \\
\hline$\leq 60$ & 78 & $46.5-147$ & & 1086 & $665.5-1918$ & \\
\hline \multicolumn{7}{|l|}{ Tumor location } \\
\hline Left lung & 79 & $43.5-147.25$ & 0.203 & 1035.5 & $661.5-1699$ & 0.150 \\
\hline Right lung & 84 & $48-167.75$ & & 1160 & $637-1847$ & \\
\hline Whole lung & 36 & $22-50$ & & 2774 & 2036-3512 & \\
\hline \multicolumn{7}{|l|}{ TNM stage } \\
\hline I and II & 56.5 & 34-76 & $<0.0001$ & 894 & $572-1586.25$ & 0.021 \\
\hline III and IV & 101.5 & $50-183.25$ & & 1182 & $692-1932.25$ & \\
\hline \multicolumn{7}{|l|}{ Lymph node metastasis } \\
\hline Yes & 94 & $50-170$ & $<0.0001$ & 1299.5 & $740.25-1994.5$ & $<0.0001$ \\
\hline No & 58 & $20.75-101.5$ & & 691.5 & $404.75-1028$ & \\
\hline \multicolumn{7}{|l|}{ Grading } \\
\hline 1 & 154 & $69.25-221$ & 0.077 & 998.5 & $587.25-3576$ & 0.892 \\
\hline 2 & 83 & $47.25-172$ & & 1043 & $636.75-2206.25$ & \\
\hline 3 & 73.5 & $43.5-131.25$ & & 1186 & $710.5-1603.75$ & \\
\hline \multicolumn{7}{|l|}{ Histological type } \\
\hline Squamous carcinoma & 78 & $48-153.5$ & 0.988 & 1098 & 725.5-2088 & 0.403 \\
\hline Adenocarcinoma & 83.5 & $42-151.5$ & & 1092 & $640.25-1739.25$ & \\
\hline Adenosquamous carcinoma & 114.4 & $32.5-237$ & & 1308.5 & $736.25-1686.5$ & \\
\hline Sarcoma $^{\mathrm{a}}$ & 74 & & & 391 & & \\
\hline
\end{tabular}

${ }^{a}$ Only one person was diagnosed with sarcoma, therefore, the value was recorded directly. VEGF, vascular endothelial growth factor; MMP-9, matrix metalloproteinase-9; NSCLC, non-small cell lung cancer.

Table III. Univariate and multivariate Cox regression analysis of inoperable NSCLC patients.

\begin{tabular}{lccc}
\hline & Univariate & \multicolumn{2}{c}{ Multivariate } \\
\cline { 2 - 4 } Variable & P-value & HR, 95\% CI & Exp (B) \\
\hline Age & 0.299 & & \\
Gender & 0.133 & $1.005-1.008$ & 1.006 \\
VEGF & $<0.0001$ & & $<0.0001$ \\
MMP-9 & 0.106 & & 1.746 \\
Histological type & 0.576 & $1.066-2.861$ & 0.027 \\
Differentiated degree & 0.232 & & \\
Lymph node metastasis & 0.001 & 0.971 & \\
Tumor location & & & \\
\hline
\end{tabular}

HR, hazard ratio; VEGF, vascular endothelial growth factor; MMP-9, matrix metalloproteinase-9; NSCLC, non-small cell lung cancer.

for MMP-9, the AUROC was 0.882 (95\% CI, 0.853-0.911). The AUROC of MMP-9 was found to be higher than that of VEGF in the various groups. The logistic regression analysis was used to estimate the diagnostic value of the two factors and the results showed that only MMP-9 remained significant $(\mathrm{P}<0.05)$ (Fig. 5A-F). 

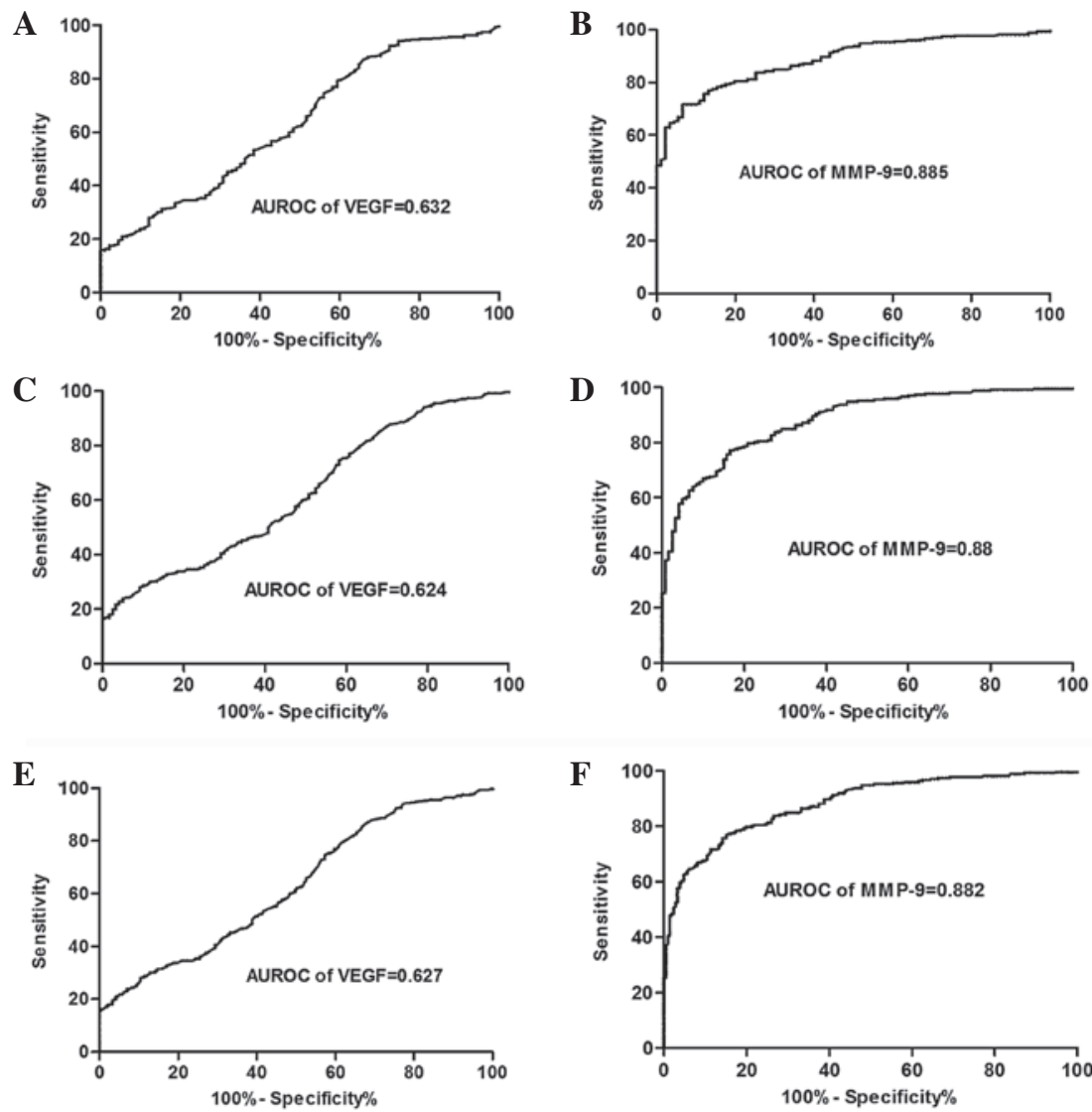

Figure 5. ROC curves of the serum levels of VEGF and MMP-9 for the diagnosis of NSCLC. (A and B) Pretreatment group was classified as the positive group and the patients with benign lung diseases as the negative group. $(\mathrm{C}$ and $\mathrm{D})$ Pretreatment group was classified as the positive group and the healthy controls as the negative group. (E and F) Pretreatment group versus the benign lung disease and healthy control groups. ROC, receiver operating characteristic; AUROC, area under the receiver operating characteristic curve; VEGF, vascular endothelial growth factor; MMP-9, matrix metalloproteinase-9; NSCLC, non-small cell lung cancer.

A

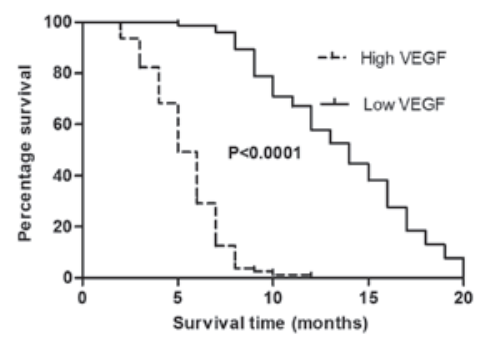

B

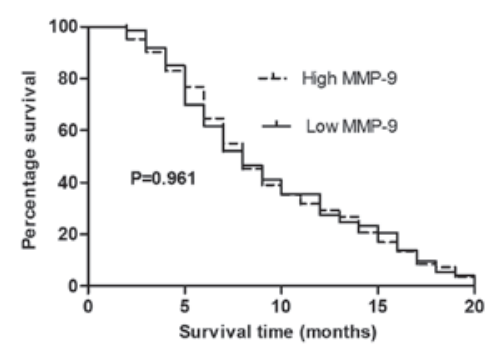

Figure 6. Kaplan-Meier survival curves. Survival curves of (A) VEGF and (B) MMP-9. VEGF, vascular endothelial growth factor; MMP-9, matrix metalloproteinase-9.

Prognostic value of VEGF and MMP-9 in the inoperable NSCLC patients. Kaplan-Meier survival curves and the log-rank test were used for statistical analysis in patients with inoperable NSCLC. Patients with low levels of VEGF $(<101.5 \mathrm{pg} / \mathrm{ml})$ were found to exhibit a significantly longer overall survival time than those with high levels of VEGF $(>101.5 \mathrm{pg} / \mathrm{ml})(\mathrm{P}<0.0001)$. However, no statistically significant difference was identified in the overall survival time between the high $(>1,182 \mathrm{ng} / \mathrm{ml})$ and low levels $(<1,182 \mathrm{ng} / \mathrm{ml})$ of MMP-9 ( $\mathrm{P}>0.05)$, as shown in Fig. 6. Cox regression models were used to analyze the effect of clinicopathological parameters on patient survival time, and the parameters included age, gender, levels of VEGF and MMP-9, histological type, grading, lymph node metastasis and tumor location (Table III). In the univariate Cox regression, lymph node metastasis $(\mathrm{P}=0.001)$ and the levels of VEGF $(\mathrm{P}<0.0001)$ in serum were found to closely correlate with patient survival time. The two variables were analyzed using multivariable Cox regression and the results indicated that lymph node metastasis $(\mathrm{P}=0.027)$ and the concentration of VEGF $(\mathrm{P}<0.0001)$ in serum remained significant. Therefore, the serum levels of VEGF and lymph node metastasis have been identified as independent prognostic factors in the inoperable NSCLC.

\section{Discussion}

Neoplasm metastasis is the leading cause of mortality in patients with lung cancer. Angiogenesis and basement membrane degradation are essential in the process of the 
growth and invasion of tumor lesions $(3,5)$. VEGF is important for angiogenesis, while MMP-9 is involved in basement membrane degradation. Recent studies have confirmed that VEGF stimulates the proliferation of endothelial cells by activating MMPs and, accordingly, inducing the angiogenesis of tumors (7,8). MMP-9 is critical in the invasion and metastasis of tumors and is also considered as a type of tumor angiogenic factor involved in the signaling system of VEGF-VEGF receptor $(9,10)$. The objective of the current study was to analyze the correlation between the serum levels of VEGF and MMP-9, as well as their diagnostic and prognostic values in NSCLC.

Compared with immunohistochemical staining, the prominent advantages of measuring angiogenic factors in the blood are high availability and reduced bias (11). Luminex multiplex assays were used to detect the levels of VEGF and MMP-9 in the serum. The serum levels of VEGF and MMP-9 were found to be significantly increased in the pretreatment group of NSCLC compared with the patients with benign lung diseases and healthy controls. These results are consistent with a number of previous observations $(12,13)$. A significant decrease in the serum levels of VEGF and MMP-9 was observed in the postoperative group compared with the pretreatment group of NSCLC patients. This result may be due to the decrease of the tumor load and suggests that the two factors are associated with the outcome of NSCLC patients.

In the present study, the serum levels of VEGF and MMP-9 were found to significantly correlate with TNM staging in the pretreatment group of NSCLC. Compared with stages I and II, the concentration of VEGF and MMP-9 in the patients with stages III and IV was significantly elevated. Currently, the expression of the two factors is detected by various methods, such as RT-PCR, immunohistochemistry and ELISA. Previous studies have shown that the expression of VEGF increases as NSCLC staging progresses (14). The expression of MMP-9 is also associated with clinicopathological stage in the serum of NSCLC (15). These hypotheses are consistent with the results of the current study. By contrast, a previous study found no correlation between the expression of the two factors and TNM staging in 91 patients with NSCLC (16).

In the present study, the serum levels of VEGF and MMP-9 in the pretreatment group of NSCLC with lymph node metastasis were significantly higher than in those without lymph node metastasis. A number of previous studies have found that the high expression of VEGF and MMP-9 closely correlate with lymph node metastasis $(15,17)$. No significant differences were identified between the levels of the two factors and age, gender, degree of differentiation, histological type and tumor location. This result is consistent with a number of previous domestic and foreign studies (16,18-20). However, a previous study reported that the expression of VEGF and MMP-9 were found to closely correlate with the degree of differentiation, using immunohistochemical detection in 136 patients with NSCLC (21). The differential expression in the serum and tissue, as well as the use of various detection methods may account for this result. All the abovementioned studies indicate that the serum levels of VEGF and MMP-9 significantly correlate with the progression and metastasis of NSCLC.

In the current study, the correlation between VEGF and MMP-9 was analyzed and the expression of the two proteins was found to positively correlate in the pretreatment group. This result may prompt a synergistic effect of the two factors in tumor angiogenesis and lymph node metastasis in the pretreatment group of NSCLC. Previous studies have detected a significant correlation between the serum levels of VEGF and MMP-9 in patients who did not undergo surgery $(7,8,22)$. However, no correlation between the serum levels of VEGF and MMP-9 was identified in the postoperative group of the current study. This may be due to the significant decrease in the concentration of the two indicators following tumor resection.

In the present study, it was found that the diagnostic accuracy of MMP-9 was improved compared with that of VEGF in the different groups. In the logistic regression models, only MMP-9 remained significant. The diagnostic values of VEGF and MMP-9 have remained unclear in previous studies $(23,24)$, but certain subsidiary diagnostic values of the two indicators function to confirm NSCLC in patients who are difficult to diagnose.

Finally, the overall survival time was analyzed in the inoperable NSCLC patients of the present study. Patients with low levels of VEGF were found to exhibit a significantly longer overall survival time than those with high levels of VEGF. A large number of previous studies have reported the prognostic value of VEGF in patients with NSCLC; a large amount of data have supported the prognostic value of the expression of VEGF and have confirmed that the overexpression of VEGF is associated with poor prognosis in NSCLC (25). By contrast, a certain study was unable to identify the prognostic value of VEGF in NSCLC using quantitative immunoassay (26).

In the current study, no statistically significant difference was identified in survival time between the high and low levels of MMP-9. A previous study indicated that there was no prognostic value of the expression of MMP-9 in 90 cases of NSCLC using immunohistochemistry (27). An additional study found no correlation between the expression of MMP-9 and the overall survival time of patients with NSCLC (16). Conversely, it has been demonstrated that MMP-9 is an independent adverse prognostic factor in the tissues and serum of lung cancer $(15,28)$. Currently, the prognostic values of VEGF and MMP-9 remain controversial.

In addition, lymph node metastasis and the pretreatment serum levels of VEGF were identified as independent prognostic variables in a multivariate analysis of the current study. Therefore, the detection of serum VEGF levels may predict the progression of tumors and aid the treatment of inoperable NSCLC patients.

Overall, the present study showed that the levels of VEGF and MMP-9 were closely correlated with the progression and metastasis of NSCLC, and that MMP-9 may be a potential diagnostic indicator of NSCLC. The pretreatment serum levels of VEGF may be an independent prognostic marker for inoperable NSCLC, and therefore may be identified as an indicator to predict the outcome of patients with inoperable NSCLC. However, there were a number of inadequacies in the current study. Firstly, the sample size was not large, therefore, future studies must be conducted in larger populations. Secondly, the follow-up was available only for inoperable patients, but not for all patients with NSCLC. All NSCLC patients must be followed up for the next five years or even decades. Finally, 
the evaluation of the expression of VEGF and MMP-9 was not evaluated in situ. An increased number of future in-depth studies are required to confirm the diagnostic and prognostic values of VEGF and MMP-9 in NSCLC.

\section{References}

1. Zhao P, Dai M, Chen W and Li N: Cancer trends in China. Jpn J Clin Oncol 40: 281-285, 2010.

2. Jemal A, Siegel R, Xu J and Ward E: Cancer statistics, 2010. CA Cancer J Clin 60: 277-300, 2010.

3. Folkman J: Tumor angiogenesis: therapeutic implications. N Engl J Med 285: 1182-1186, 1971.

4. Kerbel RS: Tumor angiogenesis. N Engl J Med 358: 2039-2049, 2008.

5. Kessenbrock K, Plaks V and Werb Z: Matrix metalloproteinases: regulators of the tumor microenvironment. Cell 141: 52-67, 2010

6. Hsu HY, Joos TO and Koga H: Multiplex microsphere-based flow cytometric platforms for protein analysis and their application in clinical proteomics-from assays to results. Electrophoresis 30: 4008-4019, 2009.

7. Hiratsuka S: Vasculogenensis, angiogenesis and special features of tumor blood vessels. Front Biosci (Landmark Ed) 16 1413-1427, 2011.

8. Zhang Y, Yu LK and Xia N: Evaluation of serum and pleural levels of endostatin and vascular epithelial growth factor in lung cancer patients with pleural effusion. Asian Pac J Trop Med 5: 239-242, 2012

9. Muramatsu M, Yamamoto S, Osawa T and Shibuya M: Vascular endothelial growth factor receptor-1 signaling promotes mobilization of macrophage lineage cells from bone marrow and stimulates solid tumor growth. Cancer Res 70: 8211-8221, 2010.

10. Hiratsuka S, Nakamura K, Iwai S, et al: MMP9 induction by vascular endothelial growth factor receptor-1 is involved in lung-specific metastasis. Cancer Cell 2: 289-300, 2002.

11. Bremnes RM, Camps C and Sirera R: Angiogenesis in non-small cell lung cancer: the prognostic impact of neoangiogenesis and the cytokines VEGF and bFGF in tumours and blood. Lung Cancer 51: 143-158, 2006.

12. Tas F, Duranyildiz D, Oguz H, Camlica H, Yasasever V and Topuz E: Serum vascular endothelial growth factor (VEGF) and bcl-2 levels in advanced stage non-small cell lung cancer. Cancer Invest 24: 576-580, 2006.

13. Jumper C, Cobos E and Lox C: Determination of the serum matrix metalloproteinase-9 (MMP-9) and tissue inhibitor of matrix metalloproteinase-1 (TIMP-1) in patients with either advanced small-cell lung cancer or non-small-cell lung cancer prior to treatment. Resp Med 98: 173-177, 2004.

14. Katsabeki-Katsafli A, Kerenidi T, Kostikas K, et al: Serum vascular endothelial growth factor is related to systemic oxidative stress in patients with lung cancer. Lung Cancer 60: 271-276, 2008
15. Zheng S, Chang Y, Hodges KB, et al: Expression of KISS1 and MMP-9 in non-small cell lung cancer and their relations to metastasis and survival. Anticancer Res 30: 713-718, 2010.

16. Yurdakul A, Akyürek N, Yilmaz Ş, Karakaya J, Memış L and Öztürk C: Prognostic impact of matrix metalloproteinases (MMP-9 and MMP-2) and vascular endothelial growth factor expression in non-small cell lung cancer. Turk J Med Sci 42: 281-288, 2012

17. Tamura M, Oda M, Matsumoto I, et al: The combination assay with circulating vascular endothelial growth factor (VEGF)-C, matrix metalloproteinase-9, and VEGF for diagnosing lymph node metastasis in patients with non-small cell lung cancer. Ann Surg Oncol 11: 928-933, 2004

18. Laack E, Köhler A, Kugler C, et al: Pretreatment serum levels of matrix metalloproteinase-9 and vascular endothelial growth factor in non-small-cell lung cancer. Ann Oncol 13: 1550-1557, 2002.

19. Shimanuki Y, Takahashi K, Cui R, Hori S, Takahashi F, Miyamoto $\mathrm{H}$ and Fukurchi Y: Role of serum vascular endothelial growth factor in the prediction of angiogenesis and prognosis for non-small cell lung cancer. Lung 183: 29-42, 2005.

20. Cox G, Jones JL and O'Byrne KJ: Matrix metalloproteinase 9 and the epidermal growth factor signal pathway in operable non-small cell lung cancer. Clin Cancer Res 6: 2349-2355, 2000.

21. Jin Y, Li JP, Tang LY, et al: Protein expression and significance of VEGF, EGFR and MMP-9 in non-small cell lung carcinomas. Asian Pac J Cancer Prev 12: 1473-1476, 2011

22. Hawinkels LJ, Zuidwijk K, Verspaget HW, et al: VEGF release by MMP-9 mediated heparansulphate cleavage induces colorectal cancer angiogenesis. Eur J Cancer 44: 1904-1913, 2008.

23. Jantus-Lewintre E, Sanmartín E, Sirera R, et al: Combined VEGF-A and VEGFR-2 concentrations in plasma: diagnostic and prognostic implications in patients with advanced NSCLC. Lung Cancer 74: 326-331, 2011.

24. Atkinson JM, Pennington CJ, Martin SW, et al: Membrane type matrix metalloproteinases (MMPs) show differential expression in non-small cell lung cancer (NSCLC) compared to normal lung: correlation of MMP-14 mRNA expression and proteolytic activity. Eur J Cancer 43: 1764-1771, 2007.

25. Farhat FS, Tfayli A, Fakhruddin N, et al: Expression, prognostic and predictive impact of VEGF and bFGF in non-small cell lung cancer. Crit Rev Oncol Hematol 84: 149-160, 2012.

26. Chakra M, Pujol JL, Lamy PJ, et al: Circulating serum vascular endothelial growth factor is not a prognostic factor of non-small cell lung cancer. J Thorac Oncol 3: 1119-1126, 2008.

27. Fujise N, Nanashima A, Taniguchi Y, et al: Prognostic impact of cathepsin $\mathrm{B}$ and matrix metalloproteinase-9 in pulmonary adenocarcinomas by immunohistochemical study. Lung Cancer 27: 19-26, 2000.

28. Martins SJ, Takagaki TY, Silva AGP, et al: Prognostic relevance of TTF-1 and MMP-9 expression in advanced lung adenocarcinoma. Lung Cancer 64: 105-109, 2009. 